\title{
Modeling the operation of mining shovel-truck systems
}

\author{
Anton Voronov ${ }^{1}$, Valeri Nesterov ${ }^{1}$, Yuri Voronov $^{2, *}$ and Artyom Voronov ${ }^{2}$ \\ ${ }^{1}$ Kuzbassrazrezugol Coal Company JSC, Kemerovo, Russian Federation \\ ${ }^{2}$ T.F. Gorbachev Kuzbass State Technical University, Kemerovo, Russian Federation
}

\begin{abstract}
Load and haul operations at open-pit mines are performed by shovel-truck systems (STSs). One of the main problems of STS is the low level of its operation quality. A means of improving the STS operation quality is its optimization, based on a systems approach, according to which the operation of system components is studied by analyzing the operation of the STS as a whole. The issues of optimal design of open-pit mining machines were being addressed. They all consider separate machines. Interpretations for mining STSs, however, have not been made. With regard to the optimization of the STS performance indicators, the systems approach consists in their optimal coordination with each other, provided that they meet the requirements for the STS operation. The optimization of the STS performance indicators is carried out according to its mathematical model, which includes the objective function and restrictions, represented in the form of linear regression analysis equations, directly linking the corresponding output performance indicators with the parameters being optimized. It is proposed to use generalized estimates as an optimization criterion, and single indicators of the STS operation quality as the parameters being optimized. This simplifies optimization and increases its accuracy, as well as provides the best degree of consistency of the parameters being optimized between themselves and the external environment.
\end{abstract}

\section{Introduction}

Currently, there are quite a large number of models that study STSs at open-pit mines [18]. However, there are not enough methodological and regulatory materials regulating such an important direction of improving the quality of objects as their optimal design. To date, several works have been devoted to the issues of optimal design of rotary drilling rigs, single-bucket hydraulic excavators and hauling equipment for open-pit mines [9]. They all look at separate machines. There are no developments with regard to mining shovel-truck systems (STSs). Nevertheless, the main methodological approaches given in [9] can be used to solve the problem of optimizing the operation of the STS as a complex of machines.

${ }^{*}$ Corresponding author: vyue.ap@,kuzstu.ru 


\section{Development of the STS design structure}

The conceptual basis for optimal design is a systems approach, which, when assigning the STS performance indicators, is that, firstly, they should correlate with each other in an optimal way, and secondly, the technical and operational indicators obtained on their basis should meet the recommended values [10].

STS as a system is characterized by structure and performance. The STS structure determines its constituent parts and connections between them and should ensure its optimal operation, for which the indicators characterizing the STS must be optimized. The structural diagram of the optimal STS design is shown in Fig. 1.

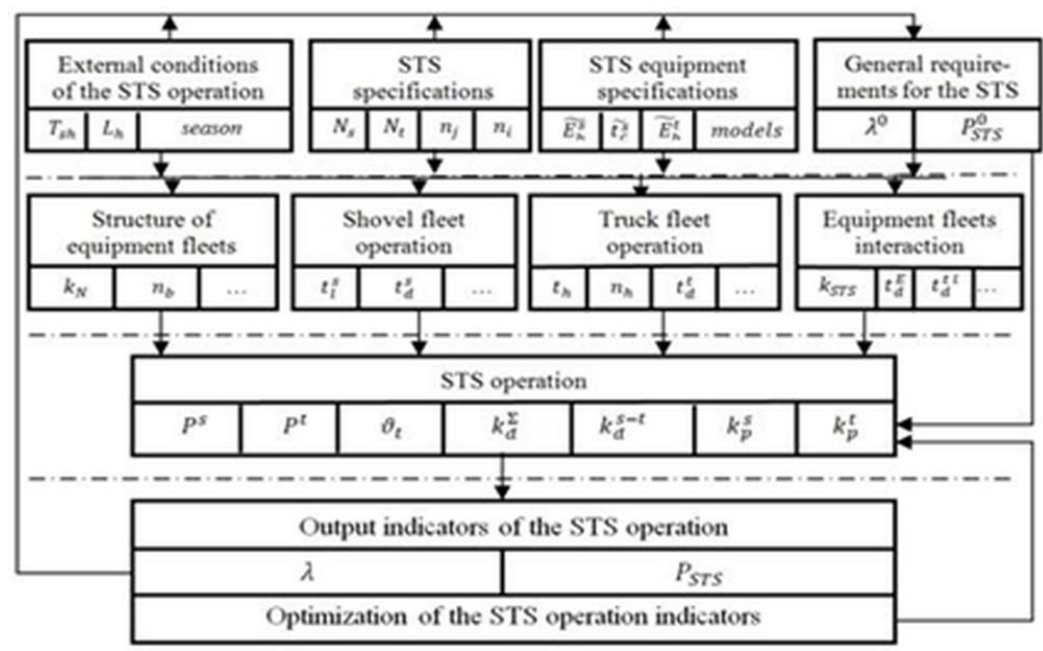

Fig. 1. Block diagram of the open-pit STS design and optimization of its operation

In Fig. 1: $T_{s h}-$ shift duration; $L_{h}-$ haul distance; $N_{s}, N_{t}, n_{j}, n_{i}$ - number of shovels, trucks and their types; $\widetilde{E_{b}^{s}}, \widetilde{t_{c}^{s}}, \widetilde{E_{b}^{t}}$ - equipment specifications; $\lambda$ - functional criterion; $P_{S T S}$ - STS performance; $k_{N}^{\prime}, k_{N}$ - coefficients of the STS structure and effective structure; $n_{b}$ - number of buckets loaded to the truck body; $t_{l}^{S}$ - truck loading time; $t_{h}$ - haul time; $n_{h}-$ number of performed hauls; $t_{d}^{s}, t_{d}^{t}, t_{d}^{E}$ - downtimes of shovels, trucks and all equipment; $t_{d}^{t l}$ - truck downtime waiting for loading; $n_{h}^{-}$- number of hauls lost due to downtimes; $k_{S T S}$ - coefficient of the STS operating performance; $P^{s}, P^{t}$ - performance of shovel and truck fleets; $\vartheta_{t}$ - operating speed of trucks; $k_{d}^{\Sigma}-$ share of downtime in the shift duration; $k_{d}^{s-t}$ - ratio of shovel and truck downtimes; $k_{p}^{s}, k_{p}^{t}$ - indicators of effective use of shovels and trucks.

It is advisable to use generalized quality assessments as an optimization criterion, and its single indicators as parameters being optimized. This makes it possible to simplify the optimization, since the problem can be divided into two simpler ones: first, optimize single quality indicators, that is, solve the problem with fewer unknowns, and then divide these optimal single indicators into more detailed ones [11]. For the calculation of single and generalized indicators of the STS operation quality, it is advisable to use the corresponding dependences of the currently widely used methodology for the non-expert assessment of the quality of mining machines [12]. 


\section{Development of models of the objective and constraints}

Models of the objective function and constraints of the mathematical optimization model (MOM) are formed as a result of the analysis of the STS operation as a system and are presented in the form of regression analysis equations that directly link the corresponding output performance indicators with the parameters being optimized.

The main output performance indicators for the STS are the functional criterion $\lambda$, as well as the STS performance $P_{S T S}$, which also determine all its other characteristics.

The functional criterion of the STS operation quality is the ratio of the operation intensities of truck and shovel fleets of the STS [13-15]. It is determined by the following formula [14]:

$$
\lambda=\frac{N_{t}}{\frac{N_{s}}{t_{l}^{S}} \cdot t_{h} \cdot k_{S T S}} .
$$

where $N_{t} / N_{s}=k_{N}^{\prime}$ - ratio of numbers of truck $\left(N_{t}\right)$ and shovel $\left(N_{s}\right)$ fleets (coefficient of the STS structure); $t_{l}^{S}, t_{h}$ - weighted averages of truck loading time and truck haul time, min.; $k_{S T S}$ - coefficient of the STS operating performance, reflecting the impact of downtime of shovel and truck fleets [13].

$$
k_{S T S}=1-\frac{\sum_{1}^{N_{s}} t_{d}^{S}+\sum_{1}^{N_{t}} t_{d}^{t}}{T_{s h}\left(N_{s}+N_{t}\right)},
$$

where $\sum_{1}^{N_{s}} t_{d}^{s}, \sum_{1}^{N_{t}} t_{d}^{t}$ - total downtimes of $N_{s}$ shovels and $N_{t}$ trucks during the shift, min.; $T_{\text {sh }}-$ shift duration, min.

The STS shift performance is determined by capabilities of the shovel fleet and can be represented as $\left(\mathrm{m}^{3} /\right.$ shift $)$ :

$$
P_{S T S}=P^{s} \cdot N_{S} \cdot T_{S h} \cdot k_{S T S}
$$

where $P^{S}$ - indicator of the shovel fleet performance, $\mathrm{m}^{3} / \mathrm{min}$. (included in the set of the STS operation quality indicators [16]).

The value $\left(N_{S} \cdot k_{S T S}\right)$ included in the formulas for determining the functional criterion (1) and the shovel fleet performance (3) is the effective number of shovels (operating without downtime), which are leading machines in the STS. Let us denote the coefficient of the effective structure of the STS truck and shovel fleets as follows:

$$
k_{N}=\frac{N_{t}}{N_{S} \cdot k_{S T S}}
$$

Then we'll obtain the following expression for the functional criterion:

$$
\lambda=\frac{k_{N} \cdot t_{l}^{S}}{t_{h}} .
$$

Having from formula (4) $N_{S} \cdot k_{S T S}=\frac{N_{t}}{k_{N}}$, and substituting it into dependence (3), we obtain: $P_{S T S}=P^{s} \cdot \frac{N_{t}}{k_{N}} \cdot T_{s h}$.

In order to avoid the need to bring together mines with significantly different numbers of truck fleets operating every shift (from 28 to 93 trucks, according to the Kuzbassrazrezugol Company), it is advisable to use the specific performance of the STS (per one truck). We have: 


$$
P_{S T S}^{s p}=\frac{P^{s} \cdot T_{s h}}{k_{N}} .
$$

The dependence of the shift duration $T_{s h}$ on the parameters being optimized will be obtained from the following considerations. Total downtime of equipment (shovels and trucks) $t_{d}^{E}=\sum_{1}^{N_{s}} t_{d}^{s}+\Sigma_{1}^{N_{t}} t_{d}^{t}$ is the total duration of shifts of all trucks minus the total haul time of all trucks during the shift, i. e.

$$
t_{d}^{E}=T_{s h} \cdot N_{t}-t_{h} \cdot n_{h}
$$

where $n_{h}$ - number of hauls performed during the shift.

On the other hand, from (2) we have:

$$
t_{d}^{E}=T_{s h}\left(N_{t}+N_{s}\right)\left(1-k_{S T S}\right) .
$$

From the equality of dependences (7) and (8), and also taking into account that $k_{S T S}=\frac{N_{t}}{N_{S} \cdot k_{N}}$, after the transformations we'll obtain:

$$
T_{s h}=\frac{t_{h} \cdot n_{h} / N_{t}}{\frac{k_{N}^{\prime}+1}{k_{N}}+\frac{1}{k_{N}^{\prime}}} .
$$

We assume that there is a relationship between the number of hauls performed by each truck during the shift $\left(n_{h} / N_{t}\right)$ and the haul duration $t_{h}$. Indeed, the longer the haul time, the fewer the number of hauls a truck can perform per shift. Calculations have confirmed the existence of such a relationship. It was obtained as a result of statistical processing of the relevant data for six mines included in the Kuzbassrazrezugol Company, and has the following form: $n_{h} / N_{t}=77,24-1,318 t_{h}$, for which the correlation coefficient is $r=0,914$; its reliability is confirmed by the Student's criterion $\left(t=4,514>t_{c r}=2,776\right)$; significance of regression parameters was confirmed by the Fisher's test $\left(F=219,48>F_{c r}=6,94\right)$.

The relationship between the indicators $k_{N}^{\prime}$ and $k_{N}$ in dependence (9) cannot be discovered, therefore, the dependence between them will be calculated through the difference $\left(k_{N}-k_{N}^{\prime}\right)$ as the weighted average value over the mines, that is, $\left(k_{N}-k_{N}^{\prime}\right)=\frac{\sum_{1}^{m}\left[\left(k_{N}-k_{N}^{\prime}\right)_{m} \cdot n_{h_{m}}\right]}{\sum_{1}^{m} n_{h_{m}}}$. Calculations have shown that the weighted average of the value $\left(k_{N}-k_{N}^{\prime}\right)$ is 0,357 , that is, $k_{N}^{\prime}=k_{N}-0,357$.

We substitute this value and the dependence for $\left(n_{h} / N_{t}\right)$ into expression (9), and we obtain the dependence of the shift duration $T_{s h}$ on two variables: the haul time of a truck $t_{h}$ and the coefficient of the effective structure of fleets $k_{N}$, and the numerator depends only on $t_{h}$, and the denominator depends on $k_{N}$.

Let us denote the numerator of the dependence (9) through the function $F_{t}$ and try to linearize it: $F_{t}=a+b t_{h}$. In this case, it was not possible to establish a linear connection between the function and the argument. Judging by the values, the function $F_{t}$ is approximately constant for any values of $t_{h}$, that is, $F_{t}=$ const. Let us define this value, as in the previous cases, as a weighted average over the mines: $F_{t}=1084,1 \mathrm{~min}$.

The value of $P^{s}$ in the dependence for determination of the specific STS performance $P_{S T S}^{S p}(6)$ is also defined as the weighted average value for the mines: $P^{s}=44,21 \mathrm{~m}^{3} / \mathrm{min}$. 
Similarly, we linearize the denominator of dependence (9): $F_{k}=k_{N}\left(\frac{k_{N}^{\prime}+1}{k_{N}}+\frac{1}{k_{N}^{\prime}}\right)=a+$ $b k_{N}$. The specified dependence has the form: $F_{k}=1,0155-0,527 k_{N}$, for which $r=$ 0,$999 ; t=44,4>t_{c r}=2,776 ; F=1492762,48>F_{c r}=6,94$.

Substituting the obtained values into the dependence for $T_{s h}(9)$, we obtain an expression for the specific STS shift performance depending on the parameters being optimized (thousand $\mathrm{m}^{3} /$ shift per 1 truck):

$$
P_{S T S}^{s p}=\frac{58,27 t_{h}\left(58,6-t_{h}\right)}{1,0155-0,527 k_{N}} .
$$

Depending on the functional criterion (5), we assume the relationship between the truck loading time $t_{l}^{S}$, and the haul time $t_{h}$. A prerequisite for this assumption is that the loading time is part of the haul time; it is only unclear how strong this connection is. The dependence has the form $t_{l}^{S}=4,978-0,0619 t_{h}$, and statistical characteristics are $r=0,792$; $t=2,597<t_{c r}=2,776 ; F=211,67>F_{c r}=6,94$.

The necessary closeness of the relationship between the indicators $t_{l}^{s}$ and $t_{h}$ according to the Student's criterion has not been confirmed, therefore, they both remain in the set of the parameters being optimized. And since there is still a statistical connection between them, since the reliability of the dependence according to Fisher's criterion is confirmed, we include this dependence to the constraints.

Thus, we get a group of three parameters being optimized, one of which $\left(k_{N}\right)$ is relative, two $\left(t_{l}^{S}\right.$ and $\left.t_{h}\right)$ are absolute. The relative parameter $k_{N}$ encompasses four absolute ones: $N_{t}$ and $N_{s}$ (the quantitative composition of truck and shovel fleets in the STS), $k_{S T S}$ (the coefficient of the STS operational performance), which, in turn, includes $t_{d}^{E}$ (total equipment downtime during the shift) and $T_{s h}$ (shift duration).

The problem of establishing the degree of influence of each of the parameters being optimized on the generalized quality indicator $k$, chosen as an optimization criterion, and forming the objective function model on this basis, refers to approximation problems and is solved using the theory of experiment planning. As a result of its solution, a polynomial regression analysis model should be obtained:

$$
k=a_{0}+a_{1} k_{N}+a_{2} t_{l}^{S}+a_{3} t_{h}
$$

reflecting the influence of each of the parameters being optimized on the optimization criterion. In expression (11) $k$ - generalized quality indicator (response function); $k_{N}, t_{l}^{S}, t_{h}-$ parameters (factors) being optimized; $a_{n}$ - coefficients.

The resulting regression dependence allows to quantify the degree of influence of each of the parameters being optimized on the generalized indicator of the STS operation quality.

When solving the problem of optimizing the STS operation quality indicators, the following restrictions are imposed.

1. Restrictions on the compliance of the actual values of the output performance indicators $\lambda, P_{S T S}$ with the prescribed values $\lambda^{0}, P_{S T S}^{0}$ (Fig. 1).

Models of these constraints can be derived from regression dependences linking the corresponding performance outputs to the parameters being optimized:

$$
\begin{gathered}
a_{10}+a_{11} k_{N}+a_{12} t_{l}^{S}+a_{13} t_{h} \geq \lambda^{0} \\
a_{20}+a_{21} k_{N}+a_{22} t_{l}^{S}+a_{23} t_{h} \geq P_{S T S}^{0} .
\end{gathered}
$$

2. Restriction on the relationship of $t_{l}^{s}$ and $t_{h}$ indicators. The relationship equation is given above. 
Since in dependences (12), the output performance indicators $\lambda^{0}$ и $P_{S T S}^{0}$, as well as the equations for the relationship of indicators $t_{l}^{S}$ and $t_{h}\left(t_{l}^{S} \div t_{h}\right)^{0}$ do not take certain values, but have a spread determined by the corresponding values of standard deviations $S_{0}^{\lambda}, S_{0}^{P S T S}$ and $S_{0}^{t}$, constraints (12) can be written as follows:

$$
\begin{gathered}
\left|\lambda^{0}-a_{10}-a_{11} k_{N}-a_{12} t_{l}^{S}-a_{13} t_{h}\right| \leq S_{0}^{\lambda} ; \\
\left|P_{S T S}^{0}-a_{20}-a_{21} k_{N}-a_{22} t_{l}^{S}-a_{23} t_{h}\right| \leq S_{0}^{P_{S T S}} ; \\
\left|\left(t_{l}^{S} \div t_{h}\right)^{0}-a_{30}-a_{31} k_{N}-a_{32} t_{l}^{S}-a_{33} t_{h}\right| \leq S_{0}^{t} .
\end{gathered}
$$

Each of these constraints can be decomposed into two constraints:

$$
\begin{aligned}
& a_{10}+a_{11} k_{N}+a_{12} t_{l}^{S}+a_{13} t_{h} \geq \lambda^{0}-S_{0}^{\lambda} ; \\
& a_{10}+a_{11} k_{N}+a_{12} t_{l}^{S}+a_{13} t_{h} \leq \lambda^{0}+S_{0}^{\lambda} ; \\
& a_{20}+a_{21} k_{N}+a_{22} t_{l}^{S}+a_{23} t_{h} \geq P_{S T S}^{0}-S_{0}^{P S T S} ; \\
& a_{20}+a_{21} k_{N}+a_{22} t_{l}^{S}+a_{23} t_{h} \leq P_{S T S}^{0}+S_{0}^{P S T S} ; \\
& a_{30}+a_{31} k_{N}+a_{32} t_{l}^{S}+a_{33} t_{h} \geq\left(t_{l}^{S} \div t_{h}\right)^{0}-S_{0}^{t} ; \\
& a_{30}+a_{31} k_{N}+a_{32} t_{l}^{S}+a_{33} t_{h} \leq\left(t_{l}^{S} \div t_{h}\right)^{0}+S_{0}^{t} .
\end{aligned}
$$

The parameters of the constraint models (13), as well as the model of the objective function (11), are established as a result of a mathematical experiment, which in this case means the calculation of the generalized indicator of the STS operation quality $k$ and the output performance indicators $\lambda, P_{S T S}^{S P}$ for certain combinations of variables according to the experiment planning matrix.

3. Boundary constraints reflecting the range of variation of the parameters being optimized. This is the constraint for their non-negativity, that is, $k_{N} \geq 0 ; t_{l}^{S} \geq 0 ; t_{h} \geq 0$.

After reducing the constraints to a convenient form, a mathematical optimization model (MOM) of the STS operation quality indicators was obtained in the following form:

$$
\begin{gathered}
k=a_{0}+a_{1} k_{N}+a_{2} t_{l}^{S}+a_{3} t_{h} \rightarrow \max ; \\
a_{11} k_{N}+a_{12} t_{l}^{s}+a_{13} t_{h} \geq \lambda^{0}-S_{0}^{\lambda}-a_{10} ; \\
a_{11} k_{N}+a_{12} t_{l}^{s}+a_{13} t_{h} \geq \lambda^{0}+S_{0}^{\lambda}-a_{10} ; \\
a_{21} k_{N}+a_{22} t_{l}^{S}+a_{23} t_{h} \geq P_{S T S}^{0}-S_{0}^{P S T S}-a_{20} ; \\
a_{21} k_{N}+a_{22} t_{l}^{S}+a_{23} t_{h} \geq P_{S T S}^{0}+S_{0}^{P S T S}-a_{20} ; \\
a_{31} k_{N}+a_{32} t_{l}^{S}+a_{33} t_{h} \geq\left(t_{l}^{S} \div t_{h}\right)^{0}-S_{0}^{t}-a_{30} ; \\
a_{31} k_{N}+a_{32} t_{l}^{S}+a_{33} t_{h} \geq\left(t_{l}^{S} \div t_{h}\right)^{0}+S_{0}^{t}-a_{30} ; \\
k_{N} \geq 0 ; t_{l}^{S} \geq 0 ; t_{h} \geq 0 .
\end{gathered}
$$

After establishing the parameters of the system (14), the MOM of the STS operation quality indicators at Kuzbass open-pit coal mines was obtained: 


$$
\begin{aligned}
& \boldsymbol{k}=-0,268+0,209 \boldsymbol{k}_{\boldsymbol{N}}+0,397 \boldsymbol{t}_{\boldsymbol{l}}^{s}-0,0415 \boldsymbol{t}_{\boldsymbol{h}} \rightarrow \boldsymbol{m a x} ; \\
& 0,130 k_{N}+0,230 t_{l}^{S}-0,0250 t_{h} \geq 0,917 ; \\
& 0,130 k_{N}+0,230 t_{l}^{s}-0,0250 t_{h} \leq 1,083 ; \\
& t_{l}^{S}+0,0619 t_{h} \geq 4,822 ; \\
& t_{l}^{s}+0,0619 t_{h} \leq 5,134 \\
& k_{N} \geq 5,124 \\
& k_{N} \leq 6,653 \\
& k_{N} \geq 0 ; t_{l}^{S} \geq 0 ; t_{h} \geq 0 .
\end{aligned}
$$

The mathematical model (15) is a linear programming problem that can be solved using standard methods.

\section{Conclusion}

Optimization of the STS operation is carried out according to its mathematical model, represented in the form of linear regression analysis equations, directly linking the output performance indicators with the parameters being optimized. It was proposed to use generalized estimates as an optimization criterion and single indicators of the STS operation quality as the parameters being optimized. This simplifies optimization and increases its accuracy, as well as provides the best degree of consistency of the parameters being optimized between themselves and the external environment.

\section{References}

1. N. Cetin, Open-pit truck/shovel haulage system simulation (Ankara, Middle East Technical University, 2004)

2. A.J. Krause, Shovel-truck cycle simulation methods in surface mining (Johannesburg, University of the Witwatersrand, 2006)

3. C.E. Stout, Simulation of a large multi-pit mining operation (Missoula, The University of Montana, 2011)

4. S. Ercelebi, A. Bascetin, J. S. Afr. I. Min. Metall. 109, 433 (2009)

5. M.A. May, Applications of queuing theory for open-pit truck-shovel haulage systems (Blacksburg, Virginia Polytechnic Institute and State University, 2012)

6. C. Burt, L. Caccetta, Interfaces. 44(2), 143 (2014)

7. S. Dindarloo, M. Osanloo, S. Frimpong, J. S. Afr. I. Min. Metall. 115, 209 (2015)

8. O. Vueykova, Obosnovanie ratsionalnoi struktury avtomobilno-ekskavatornogo kompleksa otkrytogo gornorudnogo kariera [Justification of the rational structure of the truck-shovel system at an open-pit ore mine] (Orenburg, South Ural State University, 2013)

9. Y. Voronov, Optimalnoe proektirovanie gornyh kariernyh mashin [Optimal design of open-pit mining machines] (Moscow, Innovative Engineering, 2015) (rus)

10. Y. Voronov, S. Basmanov, News of the Higher Institutions. Mining Journal. 8, 59 (2007) (rus) 
11. V. Poletaev, Osnovy upravlenija kachestvom funktsionirovanija mashin [The basics of machine quality control] (Kemerovo, Kuzbass State Technical University, 1993)

12. G. Solod, Osnovy kvalimetrii [The basics of qualimetry] (Moscow, Moscow Mining Institute, 1991)

13. Y. Voronov, Ar. Voronov, E3S Web Conf., 21 (2017)

14. Y. Voronov, A. Khoreshok, Ar. Voronov, N. Stenina, An. Voronov, Bulletin of the Kuzbass State Technical University, 3, 54 (2019)

15. Y. Voronov, An. Voronov, Ar. Voronov, E3S Web Conf., 105, 01048 (2019)

16. An. Voronov, Y. Voronov, Ar. Voronov, N. Demirel, E3S Web Conf., 105, 01058 (2019) 